\title{
Dilation-5 Embedding of 3-Dimensional Grids into Hypercubes
}

\author{
M.Y. Chan, F. Chin, C.N. Chu and W.K. Mak \\ Department of Computer Science \\ The University of Hong Kong \\ Pokfulam Road, Hong Kong
}

\begin{abstract}
We present an algorithm to map the nodes of a 3 dimensional grid to the nodes of its optimal hypercube on a one-to-one basis with dilation at most 5.
\end{abstract}

\section{Introduction}

A binary hypercube of dimension $n$, is an undirected graph of $2^{n}$ nodes labeled 0 to $2^{n}-1$ in binary where two nodes are connected if and only if their labels differ in exactly one bit position. Since a hypercube has a regular structure with a rich interconnection, it is a popular multiprocessor computer architecture. An embedding for a 3-D grid into a hypercube can be viewed as a high level description of an efficient method to simulate an algorithm designed for a parallel computer with a $3-\mathrm{D}$ grid structure on a parallel computer with a hypercube structure. Here we are interested in the problem of mapping the nodes of any 3-D grid into the nodes of its optimal hypercube (the smallest hypercube with at least as many nodes as the grid) on a one-to-one basis, so that dilation (the worst case distance between grid-neighbours in the hypercube) is bounded by a small constant.

It is known that every 2-D grid can be embedded into its optimal hypercube with at most dilation 2 [C1, C2], [HLV]. This result is optimal as it has been proven that over 38 percent of all $2-\mathrm{D}$ grids need at least dilation 2 [BS]. However, not much is known about the optimal dilation for embedding 3-D grids into optimal hypercubes. A non-trivial extension of the technique in $[\mathrm{C} 1, \mathrm{C} 2]$ for embedding $3-\mathrm{D}$ grids into optimal hypercubes with at most dilation 7 was given by Chan in [C3]. A dilation- 6 embedding scheme was derived later in $[\mathrm{LH}]$. In this paper, we introduce a simple dilation-5 embedding strategy for embedding 3 -D grids into optimal hypercubes.

\section{General Outline}

Consider a 3 -D grid G of size $\alpha \times \beta \times \gamma$. Our objective is to label each node of $\mathrm{G}$ with a unique $\left\lceil\log _{2} \alpha \beta \gamma\right\rceil$-bit binary number, which effectively names the node in the optimal $\left\lceil\log _{2} \alpha \beta \gamma\right\rceil$-cube to which it is mapped. $\mathrm{G}$ can be seen as comprising of $\gamma$ layers of 2 -D grids each of size $\alpha \times \beta$. Let $l=2^{\left\lfloor\log _{2} \alpha \beta\right\rfloor}$. To aid the assignment of binary labels, we will partition G's nodes into $l$ groups, called links, evenly in the sense that when counting from layer 0 to layer $k(0 \leq k$ $\leq \gamma-1)$, the number of nodes belonging to any particular link is either $[(k+1) \alpha \beta / l]$ or $[(k+1) \alpha \beta / l]$.

Partitioning G's nodes into $l$ links is equivalent to determining a unique pair of numbers for each node of G, namely, a link-number and a bead-number. A node's link-number indicates the link to which a node belongs, while its bead-number tells its position in that link. After the partitioning, we will use a node's linknumber to determine the first $\log _{2} l$ bits of its binary label, which we will call the link-label. And we will use a node's bead-number to determine the remaining bits of its binary label, which we will call the bead-label.

\section{Preliminaries}

In [HLV], a general embedding strategy for embedding 2-D grids into 2-D grids (of different sizes) was introduced. In particular, it can be used to embed an $\alpha \times \beta$ guest grid into an $\alpha^{\prime} \times \beta^{\prime}$ host grid where $\alpha^{\prime}={ }_{2}^{\left\lfloor\log _{2} \alpha\right\rfloor}$ and $\beta^{\prime}=\left\lceil\alpha \beta / \alpha^{\prime}\right\rceil$. (Note that in [HLV], the embedding strategy was described for embedding a $h \times w$ guest grid into a $h^{\prime} \times w^{\prime}$ host grid where $w^{\prime} \leq w$ and $h^{\prime}=\left\lceil h w / w^{\prime}\right\rceil$, here we swapped the roles of row and column for later convenience.)

As the embedding is one-one, $\alpha^{\prime} \beta^{\prime}$ has to be greater than $\alpha \beta$, and some nodes in the $\alpha^{\prime} \times \beta^{\prime}$ host grid do not correspond to any node in the $\alpha \times \beta$ guest grid. A nice property of this embedding is that any unmapped node is only found in the last column of the $\alpha^{\prime} \times \beta^{\prime}$ 
host grid. So we can view the above embedding as one that embeds an $\alpha \times \beta$ grid into a jagged grid of $\alpha^{\prime}$ rows where each row consists of either $\beta^{\prime}$ or $\beta^{\prime}-1$ nodes, and the total number of nodes of the jagged grid is exactly $\alpha \beta$. Figure 1 shows an example of such an embedding. It is proven in [HLV] that this method yields a dilation-2 embedding for any $\alpha \times \beta$ guest grid. A careful study of the proof will reveal that the method actually ensures that any two neighbouring nodes in the $\alpha \times \beta$ grid can only be mapped to one the following 5 sets of relative positions in the $\alpha^{\prime}$-row jagged grid: $\{[x, y],[x, y+1]\},\{[x, y],[x, y+2]\},\{[x, y],[x+1, y]\}$, $\{[\mathrm{x}, \mathrm{y}],[\mathrm{x}+1, \mathrm{y}-1]\},\{[\mathrm{x}, \mathrm{y}],[\mathrm{x}+1, \mathrm{y}+1]\}$ where $[x, y]$ denotes the position in row $x$, column $y$ of the jagged grid. We will utilize this 2-D grid embedding method as the first step of our embedding stratedgy, and we will refer to it as the trio's method.

The process of partitioning $\mathrm{G}$ into $l$ links depends on a length-l vector of 1 's and 2 's, $v(\alpha, \beta)[\mathrm{C} 2]$.

\section{Definition 1 Define}

$$
\begin{aligned}
v(\alpha, \beta)= & {\left[v_{0}, v_{1}, v_{2}, \ldots, v_{l-1}\right] } \\
= & {\left[\begin{array}{l}
{[\alpha \beta / l\rceil} \\
\lfloor\alpha \beta / l\rfloor \\
\lfloor 2 \alpha \beta / l\rfloor-\lfloor\alpha \beta / l\rfloor \\
\lfloor 3 \alpha \beta / l\rfloor-\lfloor 2 \alpha \beta / l\rfloor \\
\vdots \\
\lfloor(l-1) \alpha \beta / l\rfloor-\lfloor(l-2) \alpha \beta / l\rfloor
\end{array}\right] }
\end{aligned}
$$

where $l=2^{\left\lfloor\log _{2} \alpha \beta\right\rfloor}$

Basically, $v$ is defined so that the 2's are evenly distributed among the 1's when there are more 1's than 2's and vice versa when there are more 2's than 1 's. The vector $v$ has a Cyclic Sum Property which is stated below.

Definition 2 For $0 \leq s \leq l-1$ and $k \geq 0$, define

$$
\operatorname{CYCLIC-SUM}(s, k)=\sum_{i=s}^{s+k-1} v_{i \bmod l}[C 2]
$$

Fact 1 (Cyclic Sum Property)

$\lfloor k \alpha \beta / l\rfloor \leq C Y C L I C-S U M(s, k) \leq\lceil k \alpha \beta / \eta$

for $0 \leq s \leq l-1, k \geq 0$

To determine the final binary label given to each node the binary reflected Gray code sequence is used.

Definition 3 For $t \geq 0$ and $0 \leq p \leq 2^{t}-1$, define

$G R A Y(t, p)=(p+1)$ th element of the $t$-bit binary reflected Gray code sequence
For example, $\operatorname{GRAY}(3,5) \equiv 111$ since 111 is the 6 th element of $(000,001,011,010,110,111,101,100)$.

Fact 2 (Gray Code Property) In the t-bit binary reflected Gray code sequence, for any $p$ such that $0 \leq p$ $\leq 2^{t}-1$ and for any $i \geq 0$, the number of differing bits of $G R A Y(t, p)$ and $\bar{G} R A Y\left(t,(p \pm i) \bmod 2^{t}\right)$ is at most $i$.

\section{Dilation-5 Embedding Strategy}

The following steps are illustrated by Figures 1 to 4 using a $5 \times 5 \times 5$ grid as an example.

Let $l=2^{\left\lfloor\log _{2} \alpha \beta\right\rfloor}, \tilde{\alpha}=2^{\left\lfloor\log _{2} \alpha\right\rfloor}$ and $\tilde{\beta}=l / \tilde{\alpha}$.

1. Transform to $\gamma$ layers of jagged grids:

Using the trio's algorithm, transform all $\gamma$ layers of $\alpha \times \beta 2$ 2-D grids into $\gamma$ layers of identical 2-D jagged grids of $\tilde{\alpha}$ rows. (See Figure 1)

2. Partition each jagged layer into cells: Imagine there is a super-chain spanning all the nodes of a layer for each of the $\gamma$ jagged layers. Divide each super-chain, hence jagged layer, into $l$ cells according to vector $v(\alpha, \beta)$ and label the cells from 0 to $l-1$. Therefore the number of nodes in cell $i$ should be equal to $v_{i}(0 \leq i \leq l-1)$. (See Figure 2)

3. Determine the link-number of each node: For any node $\mathcal{N}$, if $\mathcal{N}$ is in cell $c(0 \leq c \leq l-1)$ of layer $k(0 \leq k \leq \gamma-1)$, its link-number is

$$
\operatorname{LINK}(\mathcal{N})=(c-k) \bmod l
$$

(See Figure 3)

4. Determine the bead-number of each node: For any node $\mathcal{N}$, if $\mathcal{N}$ is in cell $c(0 \leq c \leq l-1)$ of layer $k(0 \leq k \leq \gamma-1)$, its bead-number is

$$
\begin{aligned}
B E A D(\mathcal{N})= & C Y C L I C-S U M(\operatorname{LINK}(\mathcal{N}), k) \\
& +\delta(\mathcal{N})
\end{aligned}
$$

where $\delta$ is defined as follows: let $t=\lfloor c /(\tilde{\beta}+2)\rfloor$ if $t$ is even (odd),

$$
\delta(\mathcal{N})= \begin{cases}1 & \text { if } \mathcal{N} \text { has an immediately } \\ \text { preceding (succeeding) node } \mathcal{M} & \text { in its super-chain such that } \\ & L I N K(\mathcal{M})=L I N K(\mathcal{N}) \\ 0 & \text { otherwise }\end{cases}
$$

(See Figures 3 and 4) 
5. Determine the link-label of a node:

For any node $\mathcal{N}$, define

$L K 1(\mathcal{N})=\lfloor L I N K(\mathcal{N}) / \tilde{\beta} \mid$ and $L K 2(\mathcal{N})=$

$L I N K(\mathcal{N}) \bmod \tilde{\beta}$, the link-label of $\mathcal{N}$ is

$G R A Y\left(\log _{2} \tilde{\alpha}, L K 1(\mathcal{N})\right) G R A Y\left(\log _{2} \tilde{\beta}, L K 2(\mathcal{N})\right)$

6. Determine the bead-label of a node:

For any node $\mathcal{N}$, its bead-label is

$$
G R A Y\left(\left\{\log _{2} \alpha \beta \gamma\right\rceil-\log _{2} l, B E A D(\mathcal{N})\right)
$$

7. Concatenate the link-label and bead-label to get the complete binary label for every node.

\section{Dilation Analysis}

We will call any neighbouring nodes in the same layer of a 3-D grid G horizontal neighbours and any neighbouring nodes at the same position of 2 adjacent layers of $\mathrm{G}$ vertical neighbours.

Let us consider horizontal neighbours first.

For any horizontal neighbours $\mathcal{N}_{1}$ and $\mathcal{N}_{2}$ of the grid $G$, they must be mapped to the same jagged grid, Moreover, the trio's method will map them to one of the following 5 sets of relative positions: $\{[x, y],[x$, $y+1]\},\{[x, y],[x, y+2]\},\{[x, y],[x+1, y-1]\},\{[x, y]$, $[\mathrm{x}+1, \mathrm{y}]\},\{[\mathrm{x}, \mathrm{y}],[\mathrm{x}+1, \mathrm{y}+1]\}$.

WLOG, for Lemma 1 to Lemma 5 assume that $\mathcal{N}_{1}$ is mapped to position $[x, y]$ of a jagged layer, and $\mathcal{N}_{1}$ is in cell $p, \mathcal{N}_{2}$ is in cell $q(0 \leq p, q \leq l-1)$.

Lemma 1 If $\mathcal{N}_{2}$ is mapped to $[x, y+1]$ or $[x, y+2]$, then $p \leq q \leq p+2$.

Proof outline: Note that each cell contains 1 or 2 nodes. If $\mathcal{N}_{2}$ is mapped to $[x, y+1]$, then $p \leq q$ $\leq p+1$. If $\mathcal{N}_{2}$ mapped to $[x, y+2]$, then $p+1 \leq q$ $\leq p+2$.

Lemma 2 If $\mathcal{N}_{2}$ is mapped to $[x+1, y-1]_{2}[x+1, y]$ or $[x+1, y+1]$, then $p+\tilde{\beta}-2 \leq q \leq p+\tilde{\beta}+2$.

Proof outline: We can prove this lemma by counting the number of nodes from $\mathcal{N}_{1}$ to $\mathcal{N}_{2}$ in their superchain and using the Cyclic Sum Property of the vector $v$ used in constructing the cells.

Lemma 3 The number of differing bits of the linklabels of $\mathcal{N}_{1}$ and $\mathcal{N}_{2}$ is at most 3 if $p \leq q \leq p+2$ or $p+\tilde{\beta}-2 \leq q \leq p+\tilde{\beta}+1$, and is at most 4 if $q=$ $p+\tilde{\beta}+2$.
Proof outline: Let $q=p+i(i=0,1,2, \tilde{\beta}-2, \tilde{\beta}-$ $1, \tilde{\beta}, \tilde{\beta}+1$ or $\tilde{\beta}+2)$. It can be proved that

$$
\begin{aligned}
& L K 1\left(\mathcal{N}_{2}\right)=\left(L K 1\left(\mathcal{N}_{1}\right)+r\right) \bmod \tilde{\alpha} \\
& \text { where } r=\lfloor i / \tilde{\beta}] \text { or }[i / \tilde{\beta}] \\
& L K 2\left(\mathcal{N}_{2}\right)=\left(L K 2\left(\mathcal{N}_{1}\right)+i \bmod \tilde{\beta}\right) \bmod \tilde{\beta} \\
& \text { and } \quad\left(L K 2\left(\mathcal{N}_{1}\right)+(\tilde{\beta}-i) \bmod \tilde{\beta}\right) \bmod \tilde{\beta}
\end{aligned}
$$

Substituting $i$ and using the Gray Code Property, the results can be proved.

Lemma 4 The number of differing bits of the beadlabels of $\mathcal{N}_{1}$ and $\mathcal{N}_{2}$ is at most 2.

Proof outline: By the definition of bead-number and the Cyclic Sum Property, $\left|B E A D\left(\mathcal{N}_{1}\right)-B E A D\left(\mathcal{N}_{2}\right)\right| \leq 2$. Hence the result by the Gray Code Property.

Lemma 5 If $q=p+\tilde{\beta}+2$, the number of differing bits of the bead-labels of $\mathcal{N}_{1}$ and $\mathcal{N}_{2}$ is at most 1 .

Proof outline: Note that one of $\lfloor p /(\tilde{\beta}+2)\rfloor$ and $\lfloor q /(\tilde{\beta}+2)\rfloor$ must be odd and the other must be even. (This is the reason why the factor $\tilde{\beta}+2$ is used in defining the function $\delta$.) Then it can be proved that $\left|B E A D\left(\mathcal{N}_{1}\right)-B E A D\left(\mathcal{N}_{2}\right)\right| \leq 1$ using the Cyclic Sum Property. Hence the result by the Gray Code Property.

Now, let us consider vertical neighbours.

For the following 2 lemmas, let $\mathcal{M}$ and $\mathcal{N}$ be any vertical neighbours such that $\mathcal{M}$ is above $\mathcal{N}$. Since all layers are transformed into jagged grids in the same way, $\mathcal{M}$ and $\mathcal{N}$ will be mapped to the same position of 2 adjacent jagged grids.

Lemma 6 The number of differing bits of the linklabels of $\mathcal{M}$ and $\mathcal{N}$ is at most 2.

Proof outline: With some arithmetic manipulation, we can show that $L K 1(\mathcal{M})=L K 1(\mathcal{N}) \bmod \tilde{\alpha}$ or $(L K 1(\mathcal{N})+1) \bmod \tilde{\alpha}$ and $L K 2(\mathcal{M})=(L K 2(\mathcal{N})+$ 1) $\bmod \tilde{\beta}$. Thus the number of differing bits in both components of the link-labels of $\mathcal{M}$ and $\mathcal{N}$ are at most 1.

Lemma 7 The number of differing bits of the beadlabels of $\mathcal{M}$ and $\mathcal{N}$ is at most 2 .

Proof outline: Since $\mathcal{M}$ and $\mathcal{N}$ are mapped to the same position of 2 jagged grids, $\delta(\mathcal{M})=\delta(\mathcal{N})$. So 
$B E A D(\mathcal{N})-B E A D(\mathcal{M})=1$ or 2 . Hence the result by the Gray Code Property.

The number of differing bits of the binary labels is at most 5 for any horizontal neighbours in $\mathrm{G}$ by Lemmas 3 to 5 , and is at most 4 for any vertical neightbours by Lemmas 6 and 7 . So the strategy described gives a dilation- 5 embedding for any $3-\mathrm{D}$ grid $\mathrm{G}$.

\section{References}

[BS] J.E. Brandenburg and D.S. Scott, Embeddings of Communication Trees and Grids into Hypercubes, Intel Scientific Computers Report \#280182-001, Intel Scientific Computers, CA, 1985.

[C1] M.Y. Chan, Dilation-2 Embedding of Grids into Hypercubes, International Conference on Parallel Processing, Vol. 3, pp.295-298, 1988.

[C2] M.Y. Chan, Embedding of Grids into Optimal hypercubes, SIAM J. Comput., Vol. 20, No. 5, pp.833864, October 1991.

[C3] M.Y. Chan, Embedding of 3-Dimensional Grids into Optimal Hypercubes, Proceedings of the Fourth Conference on Hypercubes, Concurrent Computers, and Applications, pp.297-299, March 1989.

[HLV] S.H. Huang, H.F. Liu and R.M. Verma, A New Combinatorial Approach to Optimal Embeddings of Rectangles, Technical Report UH-CS-92-24. Computer Science Department, University of Houston, November 1992.

[LH] H.F. Liu and S.H. Huang, Dilation-6 Embedding of 3-Dimensional Grids into Hypercubes, in Proc. International Conference on Parallel Processing, Vol.3, pp.250-254, 1991.

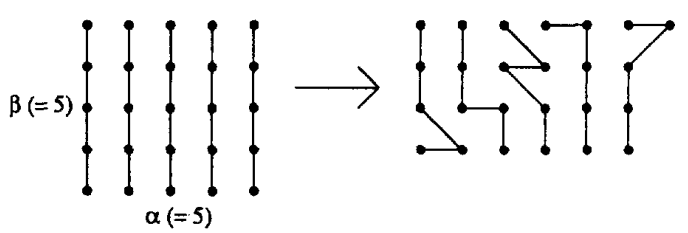

Figure 1: Transform a rectangular grid into a jagged grid by the trio's method

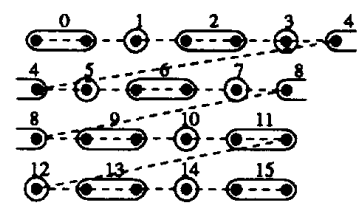

Figure 2: Partition of a jagged grid into $l$ cells. Note that the dotted line represents the super-chain and each small circle or oval represents a cell. $v(5,5)=$ $[2,1,2,1,2,1,2,1,2,2,1,2,1,2,1,2]$

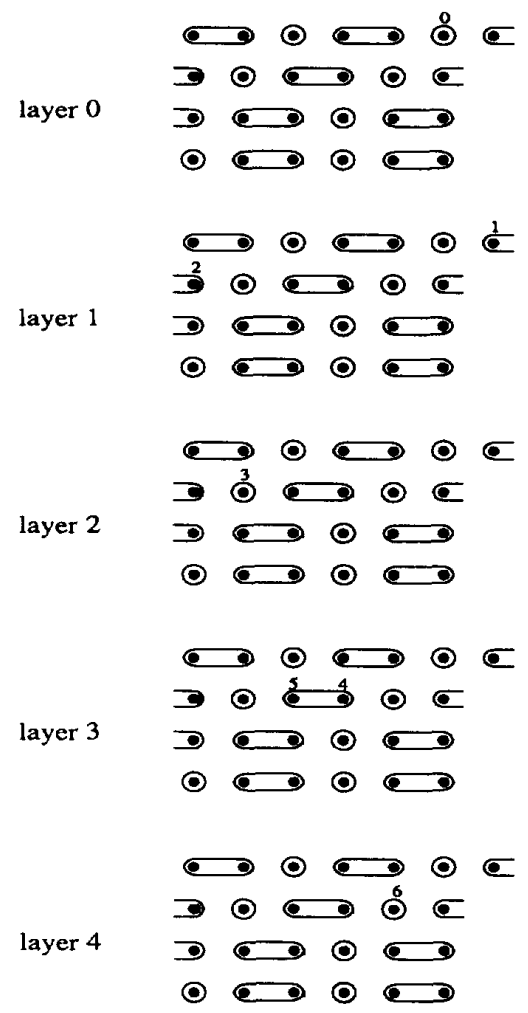

Figure 3: An example of link-number and bead-number assignments. Nodes of link 3 are shown with their beadnumbers

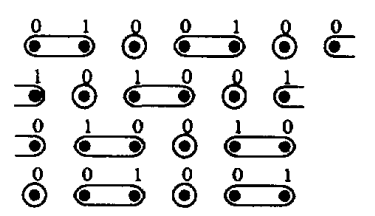

Figure 4: Values of $\delta$ function for the nodes of one layer 